\title{
Histologic Subtype
}

National Cancer Institute

\section{Source}

National Cancer Institute. Histologic Subtype. NCI Thesaurus. Code C19790.

The classification of tumors based on their histology. 